\title{
ESTUDO DE CAPACIDADE EFETIVA PARA REORGANIZAÇÃO DE SERVIÇOS EM CLÍNICA MULTIDISCIPLINAR
}

\section{EFFECTIVE CAPACITY STUDY FOR SERVICES RESTRUCTURING IN MULTIDISCIPLINAR CLINIC}

\author{
Anna Sofia Costa Neri \\ Universidade Nove de Julho - UNINOVE - SP \\ sofiaadm@hotmail.com \\ Renato Ribeiro Nogueira Ferraz \\ Universidade Mogi das Cruzes - UMC - SP \\ renatobio@hotmail.com \\ Márcia Cristina Zago Novaretti \\ Universidade Nove de Julho - UNINOVE - SP \\ mnovaretti@gmail.com
}

Submissão: $10 / 02 / 2019$

Aprovação: 28/10/2020

\begin{abstract}
RESUMO
A clínica foco deste relato foi criada para atender a crescente demanda em busca de serviços médicos e de diagnóstico em uma estrutura confortável e a preços populares. Alguns consultórios mostravam-se ociosos enquanto outros setores, como a ultrassonografia, apresentavam sobrecarga no volume de atendimentos. Tal situação sugeriu a aplicação de um estudo de capacidade efetiva para identificar a causa desta deficiência e propor soluções corretivas. Após o reconhecimento da estrutura física da clínica, foram calculadas suas capacidades instalada, disponível e efetiva. Em seguida, avaliou-se capacidade realizada por meio dos mapas de salas. Por fim, calculou-se a relação das capacidades efetiva e realizada para obtenção do índice de eficiência por sala, o que permitiu mensurar a utilização de cada sala, levando-se em consideração a ocupação. Identificou-se que capacidade efetiva da clínica era de

2.438 horas atendimento/mês, das quais apenas 1.112 horas/mês eram efetivamente utilizadas, resultando em baixo índice de eficiência (46\%). Serviços como psicologia e fonoaudiologia eram subutilizados, enquanto outros como ultrassonografia estavam sobrecarregados. Após a reestruturação e reorganização dos serviços, a ocupação passou para 1.713 horas/mês, refletindo um novo índice de eficiência, que passou a ser de $70 \%$. A implantação da proposta apresentada neste relato técnico possibilitou à clínica estudada a visualização da subutilização de suas instalações e a reorganização de serviços. As ações de intervenção para correção dos problemas incluíram desde a substituição de alguns serviços ociosos até a criação de novos departamentos e reorganização dos serviços oferecidos, com aumento do índice de eficiência e dos lucros da empresa.
\end{abstract}

Palavras-chave: Gestão em Saúde. Capacidade Instalada. Capacidade Efetiva. Produtividade. 


\begin{abstract}
The clinical focus of this report is designed to meet the growing search demand in medical and diagnostic services in a comfortable structure at popular prices. Some offices showed up idle while other sectors, such as ultrasound, had overhead in the volume of calls. This situation suggested the application of an effective capacity study to identify the deficiency cause and propose corrective solutions. After recognition of physical clinic structure, its installed, available and effective capacities were calculated. Then capacity was assessed through room maps. Finally, the ratio of actual capacity was calculated and held to obtain the index for area efficiency, allowing the use of each measuring area, taking occupancy into consideration. It was identified that effective clinical capacity was 2438 hours service / month, of which only 1112 hours / month were actually used, resulting in low efficiency (46\%). Services such as psychology and speech therapy were underutilized, while others like ultrasonography were overwhelmed. Following the restructuring and reorganization of services, occupancy increased to 1713 hours / month, reflecting a new efficiency index, which rose to $70 \%$. The implementation of the proposal presented in this technical report enabled the clinical study underutilization of view of its facilities and reorganization of services. Intervention actions to correct problems ranged from replacing some idle services to creation of new departments and offered services reorganization, increasing efficiency ratio and company's profits.
\end{abstract}

Keywords: Health Management Installed Capacity. Effective Capacity. Productivity.

\title{
1 INTRODUÇÃO
}

A Ultramed é uma clínica ambulatorial multidisciplinar particular, inaugurada no ano de 2011, com o intuito de oferecer à sociedade da cidade de Arapiraca, localizada no interior do Estado de Alagoas, e às cidades circunvizinhas, uma opção de serviços médicos e exames de diagnóstico em uma estrutura que possa proporcionar conforto e segurança, ao mesmo tempo em que oferece preços competitivos. Seus serviços são compostos por consultas médicas de diversas especialidades, atendimento odontológico, exames laboratoriais e tratamento fisioterápico, além de salas para realização de exames de imagem.

A clínica foi projetada para o atendimento de, em média, 500 pacientes por dia nas suas diversas especialidades. Entretanto, notou-se que a clínica possuía um considerável grau de ociosidade, especialmente com relação à utilização das salas para o atendimento de consultas, o que comprometia o resultado financeiro da empresa. Ao mesmo tempo em que existia esta ociosidade, observava-se que algumas especialidades apresentavam bons resultados, e até mesmo uma sobrecarga no volume de atendimentos como, por exemplo, o serviço de ultrassonografia, que utilizava $100 \%$ de sua capacidade. Assim, identificou-se a necessidade de realizar um estudo para localizar a causa desta deficiência e encontrar uma solução para correção da referida situação.

As ações propostas para identificar e corrigir o problema foram iniciadas a partir da aplicação de um estudo de capacidade efetiva, que é definida como "a capacidade que uma empresa pode esperar conseguir, considerando seu mix de produtos, métodos de programação, manutenção e padrões de qualidade" (HEIZER; RENDER, 2001). O estudo foi realizado por meio do levantamento da capacidade de produção da clínica, identificando quantos atendimentos cada sala possuía estrutura para realizar, considerando suas limitações de tempo. Com base no resultado, foi analisado o quanto a referida estrutura estava ocupada e, em seguida, foi proposta a reorganização dos serviços oferecidos de forma a aumentar a produtividade e o resultado financeiro da clínica. Basicamente, a 
reorganização levou em consideração aspectos relacionados ao tipo de serviço oferecido, à demanda observada na estrutura atual, assimcomo o retorno financeiro obtido.

Portanto, o objetivo central deste relato técnico foi apresentar a maneira pela qual o estudo de capacidade efetiva foi realizado na clínica descrita, identificando áreas ociosas e sobrecarregadas e reorganizando tais áreas, buscando aumento da produtividade e, de maneira evidente, elevando os ganhos da organização. Conforme Slack et al., (2008) " $O$ gerenciamento da capacidade está no coração das compensações entre serviço ao cliente e custos. A capacidade insuficiente deixa os clientes sem atendimento e o excesso de capacidade incorre em aumento de custos".

\section{REFERENCIAL TEÓRICO}

O termo "capacidade" pode ser entendido como "o ritmo máximo de produção de um processo" (RITZMAN; KRAJEWSKI, 2004). Para Slack; Chambers; Johhston, (2008) "o uso mais comum do termo capacidade é no sentido estático, físico de volume fixo de um recipiente ou do espaço em um edifício". Peinado; Graeml (2007), trazem o conceito de capacidade como "a ideia de competência, volume máximo ou quantidade máxima de alguma coisa".

Ao relacionarmos o termo capacidade com a atividade de produção de algum produto ou serviço, surge a necessidade de relacionar o conceito de capacidade à variável tempo. Assim, tanto Peinado; Graeml (2007), quanto Slack, Chambers, \& Johhston (2008), ressaltam a importância de conhecer a variável capacidade dentro de seu aspecto dinâmico. Para isso, é necessário associá-la à variável tempo, associação essa denominada capacidade de produção.

A capacidade de produção de uma unidade é estudada em diversos aspectos, visto que uma série de variáveis a influenciam. Assim, várias denominações foram adotadas de forma a diferenciar essas apresentações de maneira mais específica e adequada à sua aplicação no estudo da capacidade de uma unidade. Segundo Peinado; Graeml (2007), entende-se por capacidade instalada "a produção que poderia ser obtida em uma unidade fabril trabalhando 24 horas por dia, todos os dias da semana e todos os dias do mês, sem necessidade de parada, de manutenções, sem perdas por dificuldades de programação, falta de material ou outros motivos que são comuns em uma unidade produtiva". Como não é possível que uma unidade de fabricação trabalhe dentro destes moldes, esta medida é considerada apenas como base para a tomada de decisões na avaliação de ampliação da capacidade de produção.

Outra forma, mais realista, de considerar a capacidade de produção de uma unidade é através da capacidade disponível, que se conceitua como "a quantidade máxima que uma unidade produtiva pode produzir durante a jornada de trabalho disponível, sem levar em consideração qualquer tipo de perda" (PEINADO; GRAEML, 2007), a qual Slack; Chambers; Johhston, (2008) consideram como a capacidade que os técnicos tinham em mente quando projetaram a operação. Esta subdivisão traz ainda uma terceira abordagem, denominada capacidade efetiva, a qual "representa a capacidade disponível subtraindo-se as perdas planejadas desta capacidade" (PEINADO; GRAEML, 2007). Ritzman; Krajewski (2004) trazem a definição do Census Bureau para capacidade efetiva como "o maior nível de produção que uma empresa pode manter razoavelmente empregando horários de trabalho realistas dos funcionários e o equipamento atualmente instalado." As perdas consideradas nessa abordagem referem-se às perdas produtivas ocasionadas pela programação de algum evento que interrompa a produção, consideradas perdas programadas, além daquelas que não são programadas, como a falta de energia, de matériaprima ou de funcionários, por exemplo. A última forma de apresentação de capacidade a ser 
considerada é a capacidade realizada, que conforme Peinado; Graeml (2007) é obtida "subtraindo-se as perdas não planejadas da capacidade efetiva, em outras palavras, é a capacidade que realmente aconteceu em determinado período".

$\mathrm{O}$ presente trabalho busca aplicar as relações entre a capacidade efetiva e a capacidade realizada, identificando suas interações e o índice de eficiência das unidades produtivas em estudo e, com base nesse índice, avaliar o desempenho de cada unidade produtiva. Para Moraes \& Santoro (2006), eficiência "se constitui de uma medida que permite definir a capacidade real de um sistema em relação a uma capacidade teórica ou de projeto". Define-se índice de eficiência como "a relação de comparação entre a capacidade realizada e a capacidade efetiva, obtendo assim a porcentagem de eficiência da unidade produtora em realizar o trabalho programado" (PEINADO; GRAEML, 2007). Para Heizer; Render, (2001), considera- se eficiência como "uma medida da saída real sobre a capacidade efetiva".

\section{METODOLOGIA}

Considerando a necessidade da busca de informações e critérios para melhor entendimento do problema apresentado, esse estudo se caracteriza como qualitativo e exploratório, conforme define Malhotra (2005), pois esse é o modelo de pesquisa mais indicado quando se necessita explorar uma situação problema com análise de dados primários, com uma amostra pouco representativa, fornecendo ideias e informações que auxiliem o pesquisador a compreender o problema apresentado. Como estratégias de pesquisa, foram realizadas uma pesquisa bibliográfica, buscando fundamentar teoricamente os conceitos por meio de uma revisão de literatura, e uma pesquisa-ação, definida "como um tipo de investigação participante que tem como característica peculiar o propósito de ação planejada sobre os problemas detectados" (MARTINS; THEÓPHILO, 2009).

A clínica estudada encontra-se situada na cidade de Arapiraca, município localizado no estado de Alagoas. Como principal cidade do interior do estado, conta com aproximadamente

220.000 habitantes (IBGE, 2011). Reconhecida como o mais importante município do interior alagoano, Arapiraca está localizada geograficamente no centro do estado e destacase como importante centro comercial da região agreste, onde se localiza a segunda região metropolitana mais importante do estado, composta por vinte municípios, da qual Arapiraca é referência, influenciando diretamente uma população de aproximadamente meio milhão de habitantes. Localizada no centro da cidade, região de concentração do centro comercial e de facilidade de acesso ao transporte público, a clínica em avaliação neste relato técnico foi fundada com a intenção de atender a uma demanda existente na cidade, relacionada aos pacientes que não possuem planos de saúde, mas que, entretanto, não têm poder aquisitivo para pagamento de procedimentos particulares, embora possuam condições financeiras para arcar com os custos relacionados a procedimentos com valores mais acessíveis, realizando inclusive pagamento imediato. Esse tipo de negócio tem se mostrado bem competitivo na região e despertado o interesse dos profissionais de saúde que necessitam de uma estrutura para realizar seus atendimentos e, para tal, fazem parcerias com clínicas que apresentam este perfil para prestar seus serviços.

Em geral, a clínica foco do presente relato disponibiliza a estrutura física adequada e corpo profissional de apoio, que viabilizam o atendimento a seus usuários, como consultórios, salas de procedimento e de exames dentro das normas exigidas por órgãos fiscalizadores, recepcionistas para agendamento e recepção de pacientes, técnicos de enfermagem, digitadores de laudos, equipe de higienização do ambiente e segurança. Enfim, a estrutura necessária para que o profissional possa desempenhar suas atividades. 
Em contrapartida, o profissional, paga um valor de aluguel por essa estrutura, que pode ser acordado como um valor fixo ou um percentual de sua produção.

Ao observarmos as práticas de mercado, os dois modelos possuem suas vantagens e desvantagens. No primeiro caso, ao se pagar um valor fixo, a clínica tem a segurança de que receberá um valor exato que cobrirá seus custos. Entretanto, esse modelo apresenta o risco de que o profissional realize uma produção muito intensa e exija muito mais da estrutura oferecida. Nesse caso, a clínica dispenderá muito mais recursos para atendê-lo sem que tenha o retorno financeiro adequado. Ao receber participação na produção dos profissionais, a clínica desfruta a vantagem de conseguir um retorno financeiro maior, se considerarmos o aumento da produção do profissional. Tal situação mostra-se bastante atraente, visto que a intenção dos profissionais que recebem por sua produção é aumentá-la cada vez mais, já que assim seus ganhos serão maiores. Porém, um ponto muito importante a ser considerado nessa modalidade de parceria é que, em geral, os profissionais de saúde desenvolvem suas atividades em diversas instituições e, muitas vezes, o tempo por eles disponibilizado pode ser bastante limitado. Tal variedade traz a pulverização de sua demanda, o que por sua vez não garante que haverá demanda suficiente para este profissional, ou mesmo que este dê prioridade em direcionar a maior parte de seus pacientes para a clínica, já que outras relações de interesse comercial podem ocorrer junto à instituições concorrentes.

Na clínica em questão, um percentual da produção é destinado ao pagamento por produtividade, variando de acordo com o tipo de procedimento realizado. Tal modelo exige constante acompanhamento por parte dos gestores da clínica, de forma a garantir que os profissionais trabalhem em ritmo adequado, já que, caso esses profissionais desempenhem suas funções em ritmo aquém do que se espera, podem influenciar negativamente nos ganhos financeiros da empresa. Assim, a utilização do estudo de capacidade efetiva se mostrou como uma ferramenta adequada para avaliar a produtividade por sala, visto que, para avaliar a produção, a variável associada à capacidade deverá ser o tempo, que também é considerado quando se negocia a ocupação das salas pelos profissionais. Ainda, o índice de eficiência, que neste trabalho foi utilizado para avaliar a capacidade de produção de cada sala, permite uma avaliação bem objetiva dos pontos a serem trabalhados.

Para confecção deste relato foi necessário conhecer detalhadamente toda a estrutura e a forma de organização da clínica para, em seguida, identificar os pontos elencados para melhoria. Inicialmente, foi realizado o reconhecimento de sua estrutura física, que foi dividida em áreas de apoio, não menos importantes, definidas como aquelas que se prestam ao apoio das áreas onde são realizados os procedimentos e consultas, sendo estas definidas como áreas produtivas. Na clínica em questão constituíam o apoio: duas salas de espera e dois banheiros para clientes; área para funcionários composta de vestiários, banheiros e copa; área administrativa composta por almoxarifado e sala da administração; área para guarda de materiais de limpeza e descarte de lixo. As áreas produtivas foram as seguintes: oito consultórios; sala de ultrassonografia; sala de fisioterapia; sala de audiometria. Dentre os oito consultórios, um era específico para atendimento da equipe de psicologia.

Após o reconhecimento da estrutura física, foi realizado o levantamento da capacidade de produção de cada sala, considerando o horário de funcionamento da clínica e o tipo de procedimento realizado. Para tal, a variável tempo foi utilizada como principal parâmetro, visto que era utilizada para definir a forma de ocupação das salas pelos médicos prestadores de serviço, que indicavam o tempo que necessitariam da sala para realização de seus atendimentos. A clínica funcionava de segunda à sexta-feira das $7 \mathrm{~h}$ às $19 \mathrm{~h}$, e aos sábados das $8 \mathrm{~h}$ às $14 \mathrm{~h}$. Quanto ao valor do procedimento a ser levado em consideração para cálculo do percentual pago à clínica pelo profissional médico, o tempo médio de duração de cada procedimento foi estimado com base na experiência profissional dos diretores da 
própria clínica, e os valores praticados no mercado foram os utilizados para o pagamento.

Como próxima etapa, foi calculada a capacidade instalada de cada sala, considerando, conforme sua definição, a capacidade total de produção de suas instalações. Esse cálculo resultou numa capacidade produtiva total de 720 horas / mês para cada sala. Em seguida, foram calculadas as capacidades disponível e efetiva, o que permitiu identificar um total de 256 horas de capacidade disponível. No cálculo da capacidade efetiva, levando em consideração as perdas como manutenções e paradas não programadas por falta de algum recurso, foram consideradas perdas de 2 horas / mês para cada um dos oito consultórios, que possuíam estrutura mais simples e, por isso, necessitavam de interrupções menores, por exemplo, para limpeza e manutenção. Foram consideradas perdas de 3 horas / mês para as salas que possuíam equipamentos para a realização de procedimentos específicos, sendo tais horas divididas entre limpeza, manutenções preventivas e corretivas dos equipamentos de exames. Considerando tais pontos, a capacidade efetiva para as salas foi de 244 horas / mês para cada consultório e 243 horas / mês para as salas de exames.

Após os levantamentos descritos, avaliou-se capacidade realizada da clínica por meio da elaboração e preenchimento de documentos denominados mapas de salas, representados por planilhas que compreendem os dias da semana e os horários disponíveis para atendimento. Foi elaborado um mapa para cada sala, o que permitiu identificar sua real ocupação, de acordo com os horários informados previamente pelos médicos para a realização de seus atendimentos.

A avaliação da capacidade realizada foi comparada à capacidade efetiva de cada sala para obtenção do índice de eficiência por sala, com a utilização da seguinte fórmula:

\section{ÍNDICE DE EFICIÊNCIA = CAPACIDADE REALIZADA $x 100$ CAPACIDADE EFETIVA}

O cálculo do índice de eficiência permitiu mensurar a efetiva utilização de cada sala, levando-se em consideração a sua capacidade. A partir de então, foi possível identificar os pontos de ociosidade da clínica, sobre os quais foram realizadas intervenções com vistas a maximizar a produtividade da empresa, melhorando seu desempenho e, consequentemente, seus ganhos. Tais pontos foram classificados como pontos críticos, com destaque para a identificação das salas nas quais havia um excesso de demanda, ultrapassando o índice máximo de eficiência, bem como das salas subutilizadas, cujo índice de eficiência se mantinha abaixo do esperado.

\section{RESULTADOS}

O cálculo do índice de eficiência permitiu identificar uma considerável ociosidade das salas, já que identificou apenas $46 \%$ de ocupação no geral. Em uma análise pormenorizada realizada por sala, foram identificados índices de eficiência abaixo dos $10 \%$. Tal cenário apresentava como principal consequência uma considerável redução nos ganhos da clínica já que, mesmo não havendo atendimento nessas salas, e nos referidos horários, era necessário manter as estruturas de apoio na sua totalidade visando oferecer suporte como se todas as salas disponíveis estivessem ocupadas.

Para correção do problema, o primeiro passo consistiu em identificar em quais salas o índice de eficiência mostrava-se mais crítico, seja por excesso ou deficiência de ocupação. Foram identificadas cinco salas com índice de eficiência abaixo do esperado, incluindo-se a sala de fisioterapia e a sala de audiometria, as quais possuíam índice de eficiência inferior a $50 \%$, e os consultórios 3 e 5 que tinham sua ocupação em menos de $10 \%$. Outro ponto crítico era a sala de ultrassonografia, com ocupação de $100 \%$ de sua capacidade, e de onde 
partiam relatos da equipe de recepcionistas com relação à necessidade de recusar atendimento à pacientes de ultrassonografia justamente por falta de vagas em decorrência da superação da capacidade de atendimento.

Após a análise, considerando a baixa ocupação das salas destinadas à fisioterapia e à audiometria, chegou-se à conclusão que mesmo se tais setores trabalhassem em sua capacidade efetiva máxima, o retorno financeiro não seria suficiente para pagamento de seus custos junto à clínica. Quanto aos consultórios, identificou-se que os profissionais que neles atuavam não poderiam ampliar seus horários de atendimento, impedindo a realização de qualquer manobra com vistas ao aumento do índice de eficiência dos referidos setores, pelo menos com a manutenção do pessoal em atividade à época.

Diante deste cenário, as ações corretivas adotadas foram resumiram-se na concentração da produção dos consultórios 3, 4 e 5 apenas no consultório 3, elevando o índice de eficiência de $6 \%$ para $30 \%$. A utilização da estrutura do consultório 5, agora desocupado, foi utilizada para implantação dos serviços de pneumologia e broncoscopia, que contemplavam a realização de consultas e exames, o que permitiu o aumento do índice de eficiência da sala de $6 \%$ para $53 \%$. Por sua vez, o consultório 4 foi utilizado para instalação de mais uma sala de ultrassonografia, duplicando a capacidade de atendimento da clínica na referida especialidade, o que permitiu atender suprir a enorme demanda de exames de ultrassom, que não estavam sendo executados anteriormente à reorganização estrutural. Tais realocações possibilitaram à clínica melhorias em seus resultados financeiros, especialmente por que a ultrassonografia se mostrou um dos setores mais rentáveis para a organização representada neste relato,

As decisões e ações mais difíceis de serem executadas relacionaram-se aos serviços de audiometria e fisioterapia, já que, mesmo ocorrendo aumento da demanda e da oferta de atendimento, e mesmo trabalhando em sua capacidade efetiva máxima, o retorno financeiro proporcionado por tais serviços não era suficiente para custear sua presença na estrutura da clínica, especialmente em decorrência de seus reduzidos índices de eficiência. Dessa forma, optou-se pela extinção dos referidos setores. O serviço de audiometria foi substituído pela eletrocardiografia, já que uma considerável demanda por este serviço foi identificada, além do fato de que o cálculo de sua capacidade efetiva atenderia à demanda dos custos gerados pela instalação do serviço. Em continuidade, a clínica recebeu para instalação de serviço de odontologia, que substituiu o extinto setor de fisioterapia. Com o modelo de contrato sugerido, o serviço de odontologia ocuparia a grande maioria de sua capacidade efetiva, com a proposta de ocupação de $80 \%$ dos horários disponíveis.

Resumidamente, durante a realização do estudo de capacidade efetiva como método de diagnóstico para identificação de problemas relacionados à subutilização de espaços ou sobrecarga de trabalho, identificou-se que a capacidade efetiva da clínica era de 2.438 horas de atendimento / mês, das quais apenas 1.112 horas / mês eram efetivamente utilizadas, resultando em um índice baixo índice de eficiência (46\%). Após a adoção das medidas descritas, a ocupação passou para 1.713 horas por mês, o que refletiu em um índice de eficiência de $70 \%$. Considerando o número de atendimentos realizados, a clínica evoluiu de um volume médio de 3.864 atendimentos / mês para um volume estimado de 6.213 atendimentos / mês (ambos estimados pelas horas ocupadas em cada sala), o que efetivamente influenciou positivamente nos ganhos financeiros da empresa aqui avaliada.

Os números relacionados à capacidade efetiva (em horas / mês) de cada setor da clínica, ao número de atendimentos possíveis relacionados à capacidade efetiva, à capacidade realizada (em horas / mês), ao número de atendimentos efetivamente realizados de acordo com a capacidade realizada, e ao índice de eficiência da clínica avaliada, encontram-se disponíveis na Tabela 1 (antes da intervenção) e na Tabela 2 (após a 
intervenção).

Tabela 1: Capacidade efetiva e número de atendimentos possíveis, capacidade realizada e número de atendimentos realizados, e índice de eficiência de cada setor da clínica, mensurados antes da intervenção

\begin{tabular}{|l|c|c|c|c|c|}
\hline & $\begin{array}{c}\text { ANTES DA INTERVENÇĀO } \\
\text { CAPACIDADE } \\
\text { EFETIVA } \\
\text { (HORAS / MÊs) }\end{array}$ & $\begin{array}{c}\text { Número de } \\
\text { atendimentos } \\
\text { possíveis }\end{array}$ & $\begin{array}{c}\text { CAPACIDADE } \\
\text { REALIZADA } \\
\text { (HORAS / MÊS) }\end{array}$ & $\begin{array}{c}\text { Número de } \\
\text { atendimentos } \\
\text { realizados }\end{array}$ & $\begin{array}{c}\text { ÍNDICE DE } \\
\text { EFICIÊNCIA (\%) }\end{array}$ \\
\hline CONSULTÓRIO 01 & 244 & 976 & 200 & 800 & 81,97 \\
\hline CONSULTÓRIO 02 & 244 & 976 & 200 & 800 & 81,97 \\
\hline CONSULTÓRIO 03 & 244 & 976 & 16 & 64 & 6,56 \\
\hline CONSULTÓRIO 04 & 244 & 976 & 40 & 160 & 16,39 \\
\hline CONSULTÓRIO 05 & 244 & 976 & 16 & 64 & 6,56 \\
\hline FONOAUDIOLOGIA & 243 & 972 & 116 & 232 & 47,74 \\
\hline CONSULTÓRIO 07 & 244 & 976 & 40 & 160 & 16,39 \\
\hline PSICOLOGIA & 244 & 244 & 132 & 132 & 54,10 \\
\hline FISIOTERAPIA & 244 & 488 & 120 & 480 & 49,18 \\
\hline ULTRASSONOGRAFIA & 243 & 972 & 243 & 972 & 100,00 \\
\hline TOTAL GERAL & 2.438 & 8.532 & 1.123 & 3.864 & 46,06 \\
\hline
\end{tabular}

Fonte: Dados dos autores.

Tabela 2: Capacidade efetiva e número de atendimentos possíveis, capacidade realizada e número de atendimentos realizados, e índice de eficiência de cada setor da clínica, mensurados após a intervenção

\begin{tabular}{|l|c|c|c|c|c|}
\hline & $\begin{array}{c}\text { APOS A INTERVENÇA } \\
\text { CAPACIDADE } \\
\text { EFETIVA } \\
\text { (HORAS / MÊs) }\end{array}$ & $\begin{array}{c}\text { Número de } \\
\text { atendimentos } \\
\text { possíveis }\end{array}$ & $\begin{array}{c}\text { CAPACIDADE } \\
\text { REALIZADA } \\
\text { (HORAS / MÊs) }\end{array}$ & $\begin{array}{c}\text { Número de } \\
\text { atendimentos } \\
\text { realizados }\end{array}$ & $\begin{array}{c}\text { ÍNDICE DE } \\
\text { EFICIÊNCIA (\%) }\end{array}$ \\
\hline CONSULTÓRIO 01 & 244 & 976 & 200 & 800 & 81,97 \\
\hline CONSULTÓRIO 02 & 244 & 976 & 200 & 800 & 81,97 \\
\hline CONSULTÓRIO 03 & 244 & 976 & 72 & 288 & 29,51 \\
\hline BRONCOSCOPIA & 244 & 976 & 130 & 520 & 53,28 \\
\hline ULTRASSONOGRAFIA (sala 2) & 243 & 972 & 243 & 972 & 100,00 \\
\hline ELETROCARDIOGRAFIA & 243 & 972 & 130 & 520 & 53,50 \\
\hline CONSULTÓRIO 07 & 244 & 976 & 120 & 480 & 49,18 \\
\hline PSICOLOGIA & 244 & 244 & 132 & 132 & 54,10 \\
\hline ODONTOLOGIA & 243 & 729 & 243 & 729 & 100,00 \\
\hline ULTRASSONOGRAFIA (sala 1) & 243 & 972 & 243 & 972 & 100,00 \\
\hline TOTAL GERAL & 2436 & 8769 & 1713 & 6213 & 70,32 \\
\hline
\end{tabular}

Fonte: Dados dos autores.

\section{CONCLUSÕES}

A implantação da proposta de estudo de capacidade efetiva apresentada neste relato técnico possibilitou à clínica estudada a visualização da subutilização de suas instalações e a reorganização de seus serviços. As ações de intervenção para correção dos problemas aqui apresentados incluíram desde a substituição de alguns serviços ociosos até a criação de novos departamentos, reorganização dos serviços oferecidos, e consequente aumento do seu índice de eficiência, que aumentou de 46 para $70 \%$.

Os resultados positivos da proposta de intervenção aqui descrita não impedem que esta apresente algumas limitações. Uma delas refere-se ao fato de que os dados considerados para avaliação da capacidade realizada foram baseados nas horas de ocupação 
das salas e não nas horas de atendimento efetivamente realizados. A dificuldade em mensurar as horas de atendimento se deve à necessidade de uma coleta manual e diária dessas informações, o que não foi possível durante a realização do presente trabalho. Um período maior de observação após a realização da intervenção também seria necessário para eliminar a segunda limitação da pesquisa, que foi o curto período de acompanhamento após o término da reorganização. A complexidade das organizações de serviços de saúde exige um maior cuidado na implementação das estratégias de medidas de eficiência, que não contemplam a totalidade de aspectos relevantes na prestação de serviços. Por fim, a dificuldade em encontrar trabalhos disponíveis na literatura e que pudessem servir de parâmetro comparativo, enriqueceria sobremaneira a discussão dos resultados apresentados. Como proposta para continuidade do estudo, sugere-se a quantificação das horas de ocupação propostas por cada profíssional que presta serviços à clínica, possibilitando assim a obtenção de resultados mais pontuais e precisos. 


\section{REFERÊNCIAS}

HEIZER, J.; RENDER, B. Administração de Operações - Bens e Serviços -. $5^{\circ}$. ed. Rio de Janeiro: LTC, 2001.

MALHOTRA, N. K. et al. Introdução à pesquisa de marketing. São Paulo: Prentice Hall, 2005.

PEINADO, J.; GRAEML, A. R. Administração da Produção - Operações Industriais e de Serviços. Curitiba: Unicenp, 2007.

RITZMAN, L. P.; KRAJEWSKI, L. J. Administração da Produção e Operações. São Paulo: Pearson Prentice Hall, 2004.

SLACK, N. et al. Gerenciamento de Operações e de Processos. Porto Alegre: Bookman, 2008.

SLACK, N.; CHAMBERS, S.; JOHHSTON, R. Administração da Produção. $2^{\circ}$. ed. São Paulo: Atlas, 2008. 